\title{
Relationship between M100 Auditory Evoked Response and Auditory Radiation Microstructure in 16p11.2 Deletion and Duplication Carriers
}

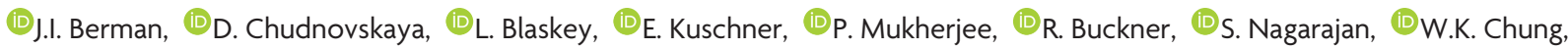

(1)E.H. Sherr, and (1)T.P.L. Roberts

\begin{abstract}
BACKGROUND AND PURPOSE: Deletion and duplication of chromosome 16p11.2 (BP4-BP5) have been associated with developmental disorders such as autism spectrum disorders, and deletion subjects exhibit a large (20-ms) delay of the auditory evoked cortical response as measured by magnetoencephalography (M100 latency). The purpose of this study was to use a multimodal approach to test whether changes in white matter microstructure are associated with delayed M100 latency.
\end{abstract}

MATERIALS AND METHODS: Thirty pediatric deletion carriers, 9 duplication carriers, and 39 control children were studied with both magnetoencephalography and diffusion MR imaging. The M100 latency and auditory system DTI measures were compared between groups and tested for correlation.

RESULTS: In controls, white matter diffusivity significantly correlated with the speed of the M100 response. However, the relationship between structure and function appeared uncoupled in 16p11.2 copy number variation carriers. The alterations to auditory system white matter microstructure in the 16p11.2 deletion only partially accounted for the 20-ms M100 delay. Although both duplication and deletion groups exhibit abnormal white matter microstructure, only the deletion group has delayed M100 latency.

CONCLUSIONS: These results indicate that gene dosage impacts factors other than white matter microstructure, which modulate conduction velocity.

ABBREVIATIONS: $\mathrm{ASD}=$ autism spectrum disorder; $\mathrm{CNV}=$ copy number variation; $\mathrm{FA}=$ fractional anisotropy; $\mathrm{HARDI}=$ high angular resolution diffusion imaging: $\mathrm{IQ}$ = intelligence quotient; $\mathrm{MD}$ = mean diffusivity; $\mathrm{MEG}$ = magnetoencephalography; $\mathrm{RD}$ = radial diffusivity; VIP = Variation in Individuals Project

R ecent studies have begun to investigate brain imaging and behavioral phenotypes in genetically defined cohorts as a way to provide better insight into etiologically heterogeneous neuropsychiatric diagnoses such as autism spectrum disorder (ASD). ${ }^{1-9}$ Deletion or duplication of the $\approx 600$ kilobase segment of chromosome 16p11.2 (bounded by BP4-BP5) has been associated with neurodevelopmental disorders, including language impairment,

Received July 28, 2015; accepted after revision November 16

From the Department of Radiology (J.I.B., D.C., L.B., E.K., T.P.L.R.), Children's Hospital of Philadelphia, Philadelphia, Pennsylvania; Department of Radiology (J.I.B., T.P.L.R.), Perelman School of Medicine, University of Pennsylvania, Philadelphia, Pennsylvania; Departments of Radiology (P.M.) and Neurology (E.H.S.), University of California, San Francisco School of Medicine, San Francisco, California; Department of Psychology (R.B.), Harvard University, Cambridge, Massachusetts; and Departments of Pediatrics and Medicine (S.N., W.K.C.), Columbia University Medical Center, New York, New York.

Paper previously presented in part at: Annual Meeting and Exhibition of the International Society for Magnetic Resonance in Medicine, May 30 -June 5, 2015;

Toronto, Ontario, Canada.

This work was supported by a grant from the Simons Foundation (SFARI 216074 and 251203) and is submitted on behalf of the Simons Variation in Individuals Project (Simons VIP) investigators. This work was supported in part by K01MH096091 and the Children's Hospital of Philadelphia/University of Pennsylvania Intellectual and Developmental Disabilities Research Center grant U54 HD086984. mild-to-moderate intellectual disability, schizophrenia, increased body mass index, epilepsy, and ASD. ${ }^{2-9}$ ASD is present in approximately $25 \%$ of individuals with a $16 \mathrm{p} 11.2$ deletion, and approximately $1 \%$ of all individuals in some cohorts with ASD have a $16 \mathrm{p} 11.2$ copy number variation $(\mathrm{CNV}){ }^{8}$

Prior studies have identified abnormal brain phenotypes associated with the 16p11.2 deletion and duplication. Increased brain size among the deletion carriers and decreased brain size among the duplication carriers were detected with structural MR imaging. ${ }^{10}$ Abnormal white matter microstructure, including increased fractional anisotropy (FA) and axial diffusivity, was observed throughout the major pathways of the cerebrum of children with 16 p11.2 deletion. ${ }^{1,12}$ The magnetoencephalography (MEG)-measured auditory evoked cortical response latency (M100) was delayed about $20 \mathrm{~ms}$ in children with 16p11.2 deletion compared with matched controls, but not in children with

Please address correspondence to Jeffrey I. Berman, MD, Children's Hospital of Philadelphia, Department of Radiology, 34th and Civic Center Blvd, Philadelphia, PA 19104; e-mail: bermanj@email.chop.edu

- Indicates open access to non-subscribers at www.ajnr.org

http://dx.doi.org/10.3174/ajnr.A4687 
16p11.2 duplication. ${ }^{13}$ The purpose of this study was to determine the role of abnormal thalamocortical white matter microstructure in modulating the profoundly delayed M100 auditory evoked response.

Abnormal structure and function of the auditory system and superior temporal gyrus have previously been associated with ASD, motivating this study of $16 \mathrm{p} 11.2 \mathrm{CNV} .{ }^{14,15}$ Diffusion MR imaging is sensitive to white matter architecture, and high angular resolution diffusion imaging (HARDI) fiber tractography is used to delineate the $3 \mathrm{D}$ position of the auditory radiation bilaterally. ${ }^{16}$ The MEG-derived M100 evoked field component is generated by neurons in the superior temporal gyrus in response to an auditory stimulus. The latency of the M100 provides a measure of white matter conduction and synaptic transmission speed integrated from the ear to the cortex as well as corticocortical processing in primary and association auditory cortices. This study combined MEG and diffusion MR imaging in a group of subjects with a defined genetic etiology. We hypothesized that the relationship between structure and function is altered in children with $16 \mathrm{p} 11.2$ deletion and duplication.

\section{MATERIALS AND METHODS \\ Participants}

Deletion carriers were recruited through the Simons Variation in Individuals Project (VIP, https://clinicaltrials.gov/ct2/show/ NCT01238250) Connect Web site (https://www.simonsvipconnect. org/). ${ }^{1}$ The $16 \mathrm{p} 11.2$ deletion participants were identified by clinical chromosome microarrays and included individuals with the same recurrent $\sim 600-\mathrm{kb}$ deletion (chr16:29, 652,99030,199,351; hg19) without other known genetic diagnoses or pathogenic CNVs. Age-matched neurotypical participants had a chromosome microarray to rule out abnormal CNVs at the 16 p11.2 locus or elsewhere in the genome. Exclusion criteria included the inability to speak English fluently, drug use, or significant structural abnormalities on MR imaging. Any control subject with a potential clinical finding on MR imaging was excluded. Any deletion or duplication carrier with a clinical finding on MR imaging unrelated to $16 \mathrm{p} 11.2$ or an overt structural anomaly such as agenesis of the corpus callosum or polymicrogyria was also excluded. All children in the typically developing control group had no neurologic or genetic disorder and no evidence of current or past psychiatric diagnosis as measured by a parent report of Diagnostic and Statistical Manual of Mental Disorders, 4th ed, symptoms on a structured diagnostic interview, the Diagnostic Interview Schedule for Children, Version IV.

Participants were administered psychological and diagnostic measures by experienced and licensed child psychologists. Cognitive and language measures used for the current analyses included the Clinical Evaluation of Language Fundamentals, 4th ed, as a measure of basic language functioning, and the Differential Ability Scales, Special Nonverbal Composite, 2nd ed, as a measure of nonverbal intellectual functioning. ${ }^{17,18}$ Clinical Diagnostic and Statistical Manual of Mental Disorders, Text Revision, 4th ed, diagnoses were obtained by using all information gathered during the evaluation. Autism diagnostic measures included the Autism Diagnostic Observation Schedule and the Autism Diagnostic Interview, Revised. ${ }^{19,20}$

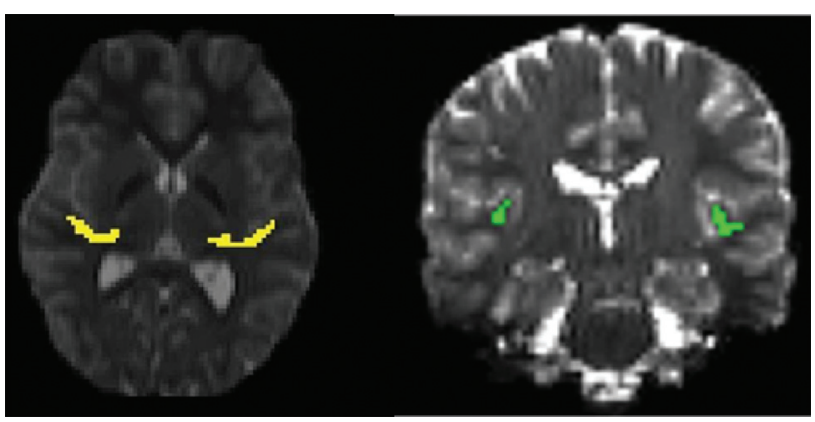

FIG 1. ROls for diffusion MR imaging measures are shown. Left: Axial section through the auditory radiation, as defined by HARDI tractography. Right: Coronal section through the transverse gyrus (Heschl's gyrus) ROI as defined by the white matter parcellation performed by FreeSurfer.

\section{MR Imaging}

Imaging was performed at either the Children's Hospital of Philadelphia or the University of California with a 3T Tim Trio scanner (Siemens, Erlangen, Germany), a 32-channel radiofrequency head coil, and identical pulse sequences. The intersite equivalency of image data quality was confirmed before the study by scanning 5 subjects at both sites, and image quality was monitored during the study with phantom quality-assurance studies. ${ }^{21}$

The whole-brain DTI acquisition used 30 diffusion gradient directions at $b=1000 \mathrm{~s} / \mathrm{mm}^{2}, 1 b=0 \mathrm{~s} / \mathrm{mm}^{2}$ volume, TR/TE $=10$ $\mathrm{s} / 80 \mathrm{~ms}$, voxel size $=2 \times 2 \times 2 \mathrm{~mm}$, and a $128 \times 128$ matrix. A separate whole-brain HARDI acquisition used 64 gradient directions at $b=3000 \mathrm{~s} / \mathrm{mm}^{2}, 2 b=0 \mathrm{~s} / \mathrm{mm}^{2}$ volumes, TR/TE $=13.9$ $\mathrm{s} / 119 \mathrm{~ms}$, voxel size $=2 \times 2 \times 2 \mathrm{~mm}$, and a $128 \times 128$ matrix. Both diffusion acquisitions used a Stejskal-Tanner monopolar, spin-echo echo-planar sequence, a parallel acceleration factor of 2 , and axial sections. Structural imaging was performed with a T1-weighted multiecho 3D magnetization-prepared rapid acquisition of gradient echo and $\mathrm{TE}_{\text {minimum }}=1.64 \mathrm{~ms}, \mathrm{TR}=$ $2530 \mathrm{~ms}$, TI $=1200 \mathrm{~ms}$, flip angle $=7^{\circ}$, and $1-\mathrm{mm}$ isotropic resolution. ${ }^{22}$

Probabilistic tractography by using the solid angle Q-ball imaging HARDI reconstruction was used to delineate the auditory radiation from the thalamus to the auditory cortex. ${ }^{16,23}$ Additional ROIs just in the transverse gyri (Heschl's gyrus) were defined from the FreeSurfer (http://surfer.nmr.mgh.harvard.edu) white matter parcellation (Fig 1). ${ }^{24}$ The $b=1000 \mathrm{~s} / \mathrm{mm}^{2}$ DTI data were used for all microstructural measures.

\section{Magnetoencephalography}

Data were acquired by using a similar 275-channel whole-head CTF magnetometer at either imaging site. The auditory stimulus consisted of passively presented tones of 200, 300, 500, and 1000 $\mathrm{Hz}$ of 300-ms duration (10-ms ramps) repeated 130 times each. The interstimulus interval varied randomly between 900 and 1100 ms. The M100 response in the left and right superior temporal gyrus was detected with a standard dipole source model that transforms the MEG sensor signals into source (brain) space. The details of the source modeling procedure have been previously described. ${ }^{13}$

AJNR Am J Neuroradiol 37:1178-84 Jun 2016 www.ajnr.org 1179 


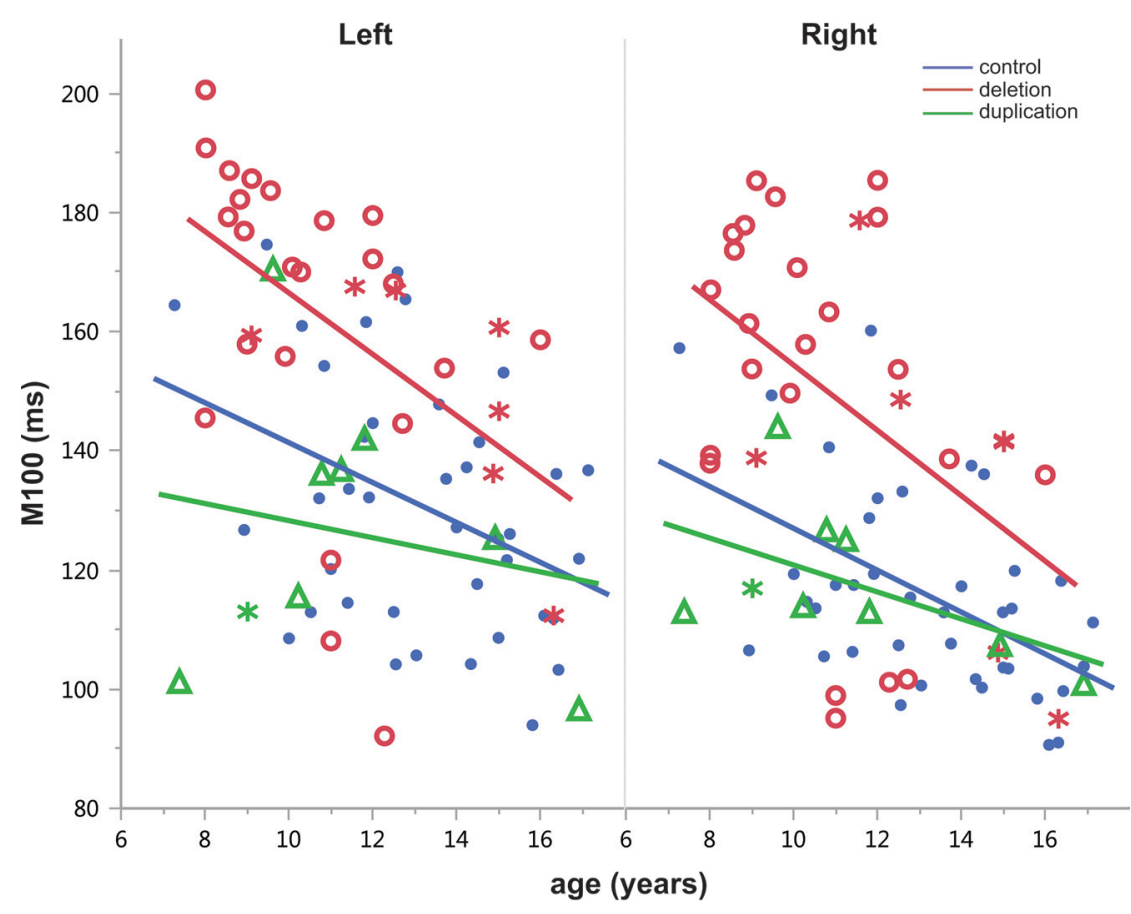

FIG 2. Developmental trajectory of M100 versus age for the left and right hemispheres. Left and right control and deletion regression lines significantly decrease with age $(P<.001$ each). There is no significant difference in slope between the groups. Individuals with an ASD diagnosis are indicated by an asterisk. The elevated M100 latency in the deletion group can be observed in both hemispheres.
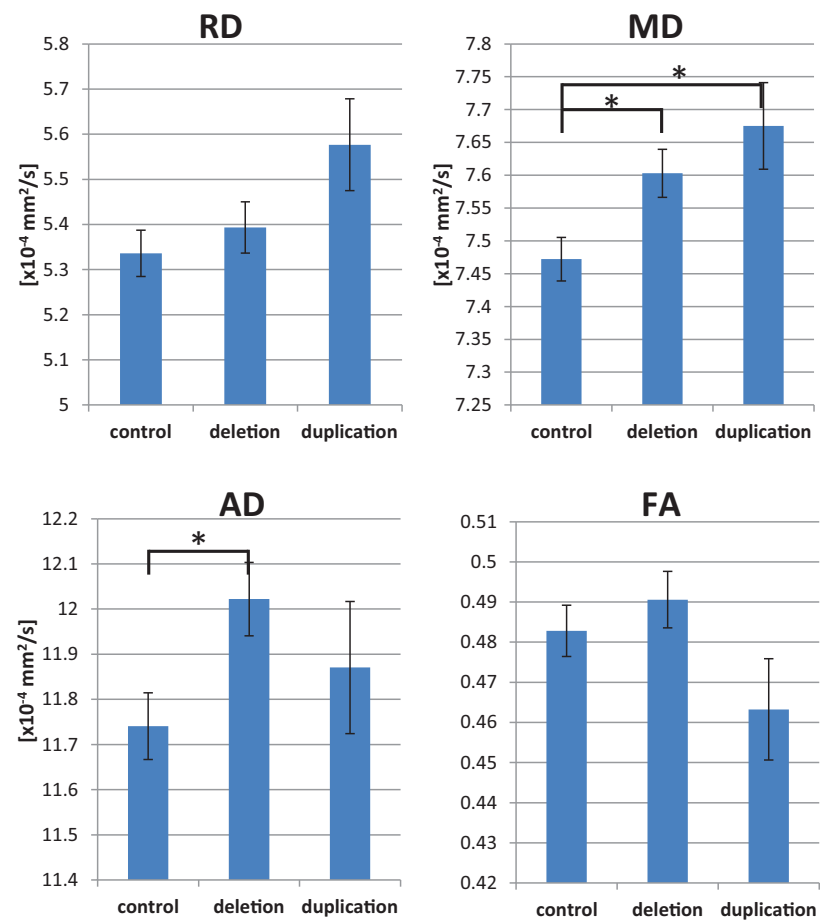

FIG 3. Group comparisons of auditory radiation DTI parameters are shown. The deletion group exhibits elevated $A D$, and both $C N V$ groups have elevated MD. The asterisk indicates $P<.05 ; A D$, axial diffusivity.

\section{Statistical Analysis}

Statistical analysis was performed by using SPSS (Version 21; IBM, Armonk, New York) and JMP (Version 11; SAS Institute, Cary, North Carolina) with a threshold for significance at $P=.05$.
M100 latency varies by hemisphere and stimulus tone frequency ${ }^{25}$; however, a single "effective" M100 for each subject's left and right hemisphere was calculated with a linear mixed-model. The model contains fixed effects of hemisphere, stimulus frequency, group, imaging site, and age and a random effect of subject. Fitted values from the model were used to predict M100 values for subjects with partially missing observations.

For analysis of M100 and DTI group differences, a linear regression was performed with effects of group, age, hemisphere, imaging site, and nonverbal intelligence quotient (IQ). The Dunnett method for multiple comparisons was used to compare deletion and duplication carrier groups with the control group. For multimodal analysis, multivariable linear regression was performed with M100 as the dependent variable and the effects of DTI parameter, group, hemisphere, nonverbal IQ, and imaging site. The interaction between DTI parameter and group was added to the model to test for group differences in the regression slope. Data were segmented by group and hemisphere for additional analyses.

\section{RESULTS}

\section{Population}

This study included 78 children imaged at either Children's Hospital of Philadelphia $(n=34)$ or the University of California $(n=$ 44). Thirty pediatric deletion carriers, 9 duplication carriers, and 39 typically developing children were included. The mean age of deletion carriers was $11.2 \pm 2.5$ years (range, $8-16$ years) with 17 males and 13 females. The mean age of duplication carriers was $11.3 \pm 3$ years (range, $7-16$ years) with 6 males and 3 females. The mean age of control children was $13.2 \pm 2.4$ (range, $7-17$ ) with 24 males and 15 females. There was a significant difference in chronologic age between the control and deletion groups $(P=$ .006; Tukey honest significance test) but no significant difference in age between control and duplication groups or between deletion and duplication groups $(P=.13, P=.99$, respectively). Age was included as a covariate in the group comparisons of DTI metrics and M100 to account for the differences in age. There was no significant group difference in the sex ratio $(P=.85$; Fisher exact test). MEG data from some of these individuals has been independently reported in Jenkins et al. ${ }^{13}$ Diffusion MR imaging findings from a subset of these subjects have been reported in Berman et al. ${ }^{12}$

Mean nonverbal IQ of the control group (mean, 107.6 \pm 13.1 ) was significantly higher than that in the deletion group (mean, $91.7 \pm 15.5)$ and the duplication group (mean, 81.4 \pm 12.1$)(P<$ .001 , each Tukey honest significance test). Nonverbal IQ was not significantly different between duplication and deletion groups 
$(P=.14)$. Seven deletion carriers and 1 duplication carrier met the diagnostic criteria for ASD.

\section{M100 Latency}

As previously reported, ${ }^{13}$ the deletion group exhibited significantly prolonged M100 latencies with a group difference of 20.6 $\mathrm{ms}(P<.001)$. There was no group difference between duplication and control M100 latencies. M100 latency was significantly longer in the left hemisphere by approximately $6.6 \mathrm{~ms}(P<.001)$. M100 was observed to decrease with age at a rate of about $4 \mathrm{~ms}$ per year $(P<.001)$. When analyzed separately by hemisphere and group, M100 significantly decreased in the left and right hemispheres of the control and deletion groups $(P<.001$ each, Fig 2$)$. There was no significant group difference in the rate of M100 maturation. Imaging site and nonverbal IQ were not significant predictors of M100 latency.
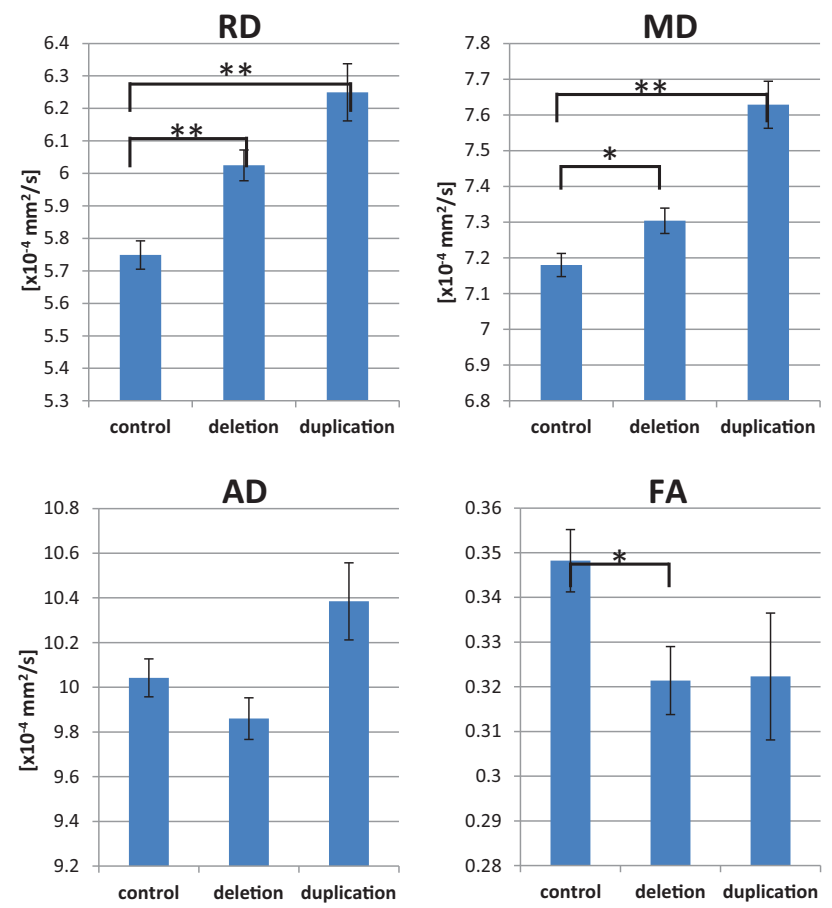

FIG 4. Group comparisons of Heschl's gyrus DTI parameters are shown. The deletion group exhibits elevated RD and MD and decreased FA. The duplication group exhibits higher MD and RD along with a trend toward decreased FA. The asterisk indicates $P<.05$; double asterisks, $P<.001 ; A D$, axial diffusivity.

Correlation of DTI with M100 within groups ${ }^{\mathrm{a}}$

\begin{tabular}{|c|c|c|c|c|c|c|}
\hline & \multicolumn{3}{|c|}{ Auditory Radiation } & \multicolumn{3}{|c|}{ Heschl's Gyrus } \\
\hline & Control & Deletion & Duplication & Control & Deletion & Duplication \\
\hline \multirow[t]{2}{*}{ MD } & 16.4 & 14.7 & 1.1 & 29.9 & 1.2 & 24.7 \\
\hline & $P=.044^{b}$ & $P=.3$ & $P=.9$ & $P<.001^{b}$ & $P=.9$ & $P=.2$ \\
\hline \multirow[t]{2}{*}{ FA } & -67.4 & 73.2 & -43 & -78.3 & -111 & -75.2 \\
\hline & $P=.15$ & $P=.3$ & $P=.7$ & $P=.063$ & $P=.2$ & $P=.4$ \\
\hline \multirow[t]{2}{*}{ RD } & 10.6 & 1.3 & 2.3 & 21.7 & 12.8 & 17.4 \\
\hline & $P=.048^{b}$ & $P=.9$ & $P=.9$ & $P<.001^{\mathrm{b}}$ & $P=.3$ & $P=.2$ \\
\hline \multirow[t]{2}{*}{$A D$} & 0.6 & 11.7 & -1.8 & 2.4 & -4.9 & 1.9 \\
\hline & $P=.9$ & $P=.11$ & $P=.8$ & $P=.5$ & $P=.4$ & $P=.8$ \\
\hline
\end{tabular}

Note:-AD indicates axial diffusivity.

${ }^{a}$ Regression coefficients by group are shown with $P$ values. Coefficients indicate the number of milliseconds per variable unit. MD, RD, and $A D$ have units of $\times 10^{-4} \mathrm{~mm}^{2} / \mathrm{s}$.

bignificant.

\section{DTI Metrics}

As previously reported, the auditory radiation of the deletion group had abnormally high mean diffusivity $(\mathrm{MD})(P=.027)$, which was primarily driven by an increase in axial diffusivity $(P=$ .033 , Fig 3 ). The duplication group had elevated auditory radiation mean diffusivity $(P=.021)$, which was driven by trends of higher radial diffusivity (RD) and axial diffusivity.

Within Heschl's gyrus, the deletion group exhibited significantly elevated $\mathrm{MD}$ and $\mathrm{RD}(P=.033, P<.001$, respectively) along with significantly decreased FA $(P=.031$, Fig 4$)$. The duplication group exhibited higher Heschl's gyrus MD and RD $(P<$ .001 each) and a trend toward decreased FA. Decreasing MD with age was observed in the left and right hemispheres of the control and deletion groups $(P<.05$ each $)$.

\section{Multimodal Analysis}

Overall across groups, there was a significant main effect of auditory radiation $\mathrm{MD}(P=.048)$ and Heschl's gyrus FA $(P=.023)$, $\operatorname{MD}(P=.011)$, and RD $(P<.001)$ on M100 latency (with elevated $\mathrm{MD}$ or RD and decreased FA predicting longer latencies). Interactions between DTI parameters and group were not significant. Post hoc analysis within groups of relationships between auditory system white matter microstructure and M100 latency is shown in the Table. In controls, M100 latency was positively correlated with both $\mathrm{MD}$ and $\mathrm{RD}$ in the auditory radiation and $\mathrm{He}-$ schl's gyrus ROI. A trend toward an inverse correlation of Heschl's gyrus FA and M100 was observed in controls $(P=.063)$. No significant correlations between DTI parameters and M100 latency were observed among the deletion or duplication groups.

Correlations between DTI parameters and M100 latency were separately examined in the left and right hemispheres for each group. The deletion and duplication groups exhibited no significant correlations with M100 in either the right or left hemispheres. Left hemispheric DTI parameters were not significant predictors of M100 in controls. In controls, right hemisphere microstructure and M100 latency were more strongly coupled than in the left hemisphere. Right hemisphere MD and RD were significantly correlated with M100 latency in both the auditory radiation and transverse gyrus of controls $(P<.05$, Figs 5 and 6$)$. Although the correlations between DTI parameters and M100 were not significant in the right hemisphere of 16 p11.2 deletion carriers, the regression slopes were not significantly different between groups. The slopes describing the relationship between right hemisphere control auditory radiation MD and RD with M100 were observed to be similar to those in the deletion group (Figs 5 and 6). Deletion and duplication carriers with an ASD diagnosis are indicated in Figs 5 and 6. The small subsets of deletion or duplication carriers with an ASD diagnosis were not overtly different from those without an ASD diagnosis.

\section{DISCUSSION}

In controls, the degree of white matter myelination, axonal density, and integrity as measured with DTI were ob- 

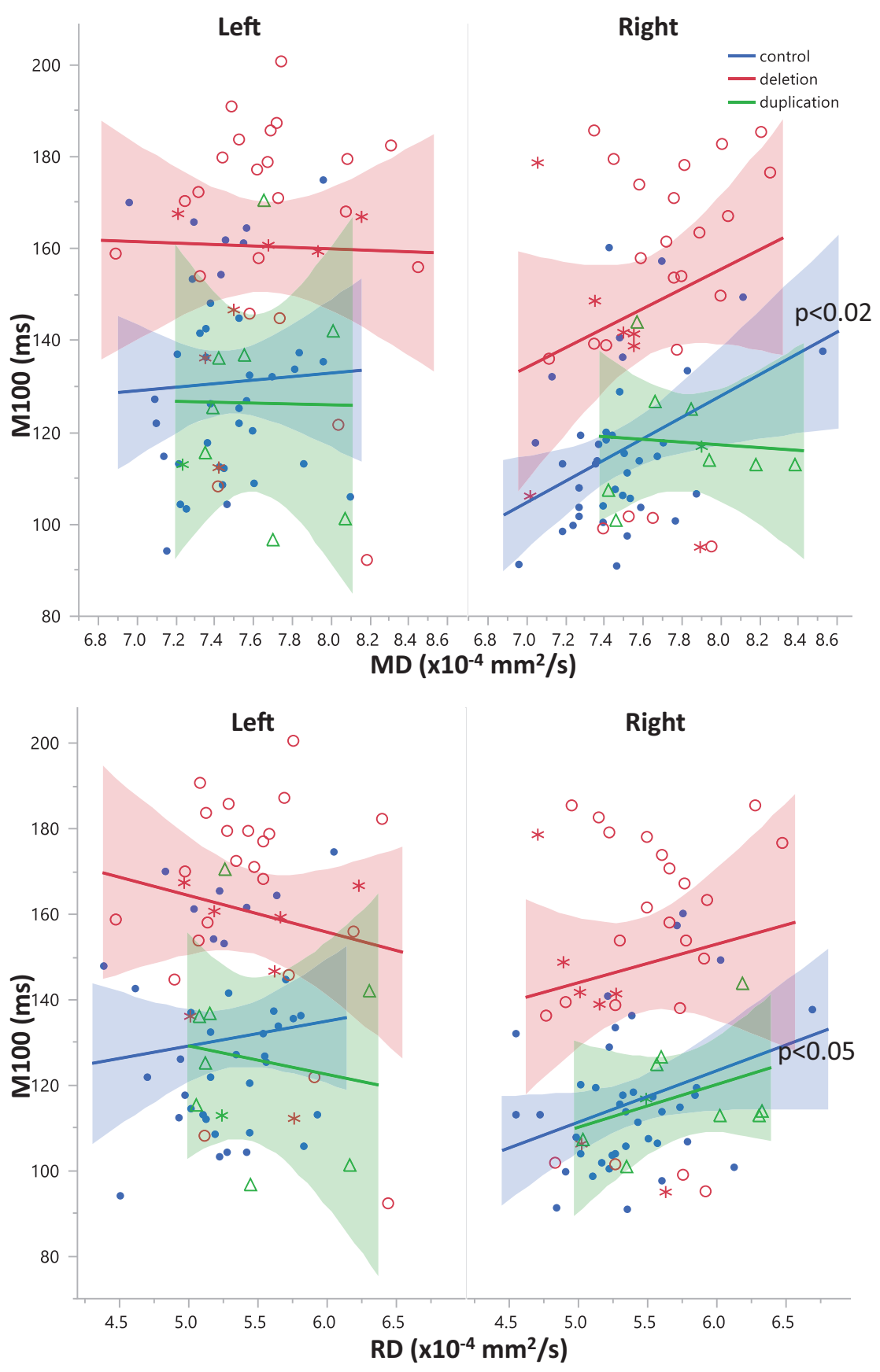

FIG 5. Relationships between diffusivity and $M 100$ are shown for left and right auditory radiations. While overall there is a significant main effect $(P<.05)$ of MD on M100 latency, post hoc analysis within groups reveals that this is only significantly resolved for right hemisphere control $\mathrm{MD}$ and $\operatorname{RD}(P=.017$ and $P=.049$, respectively). Control subjects are blue, subjects with deletion are red, and subjects with duplication are green. Subjects with deletion and duplication with an ASD diagnosis are indicated by asterisks.

served to significantly modulate the speed of the M100 response. The relationship between structure and function appears uncoupled in 16p11.2 CNV carriers; however, the slope of the relationship still appears similar to that of controls. Both duplication and deletion groups exhibited white matter microstructural abnormalities, but M100 latency was delayed only in the deletion group. These results indicate that gene dosage impacts white matter microstructure and other factors that modulate conduction velocity. A limitation of the current study is the small number of subjects with ASD; however, no overt trends differentiating $16 \mathrm{p} 11.2 \mathrm{CNV}$ subjects with and without ASD diagnosis were observed.

In addition to the previously reported large (20-ms) M100 latency delay in the deletion group, we additionally report a significant maturational decrease of the M100 of control and deletion groups. ${ }^{13}$ The duplication group also exhibited shortening of the M100 latency with age, but the relationship did not reach significance because of the small study population. Both deletion and duplication groups exhibited abnormal patterns of diffusivity in the thalamocortical white matter. Because of the relatively low number of subjects with duplication, it is difficult to determine whether thalamocortical white matter is more severely altered in deletion or duplication carriers. The unusual increase in axial diffusivity of the thalamocortical white matter is consistent with prior work using Tract-Based Spatial Statistics to examine white matter in 16p11.2 deletion. ${ }^{11}$ Most interesting, the 16p11.2 duplication group has normal or possibly shorter M100 latency in the presence of microstructural abnormalities.

Overall, there was a significant association between microstructural parameters (MD and RD) and M100 latency, with elevated diffusivity predicting longer latency. However, within-group analyses indicated that the relationship is significant only in the control group and only in the right hemisphere. A prior study similarly observed a degraded relationship between the MEG 50-ms auditory evoked response and FA in children with ASD. ${ }^{14}$ We report that the M100 versus MD and RD slopes in controls were not significantly different from the slopes in 16p11.2 CNVs. The similarity of the structure-function slope between controls and deletion carriers indicates that white matter still modulates M100 latency, though it has a diminished role. The degraded relationship between structure and function in 16p11.2 $\mathrm{CNV}$ and the difference in regression intercepts, despite similar slopes, indicate the influence of factors not accounted for by DTI measurements alone.

This study determined that the alterations to auditory system white matter structure are insufficient to completely explain the large delay in M100 latency in deletion carriers. Given the MDto-M100 slope measured in this study, a $0.8 \times 10^{-4} \mathrm{~mm}^{2} / \mathrm{s}$ in- 

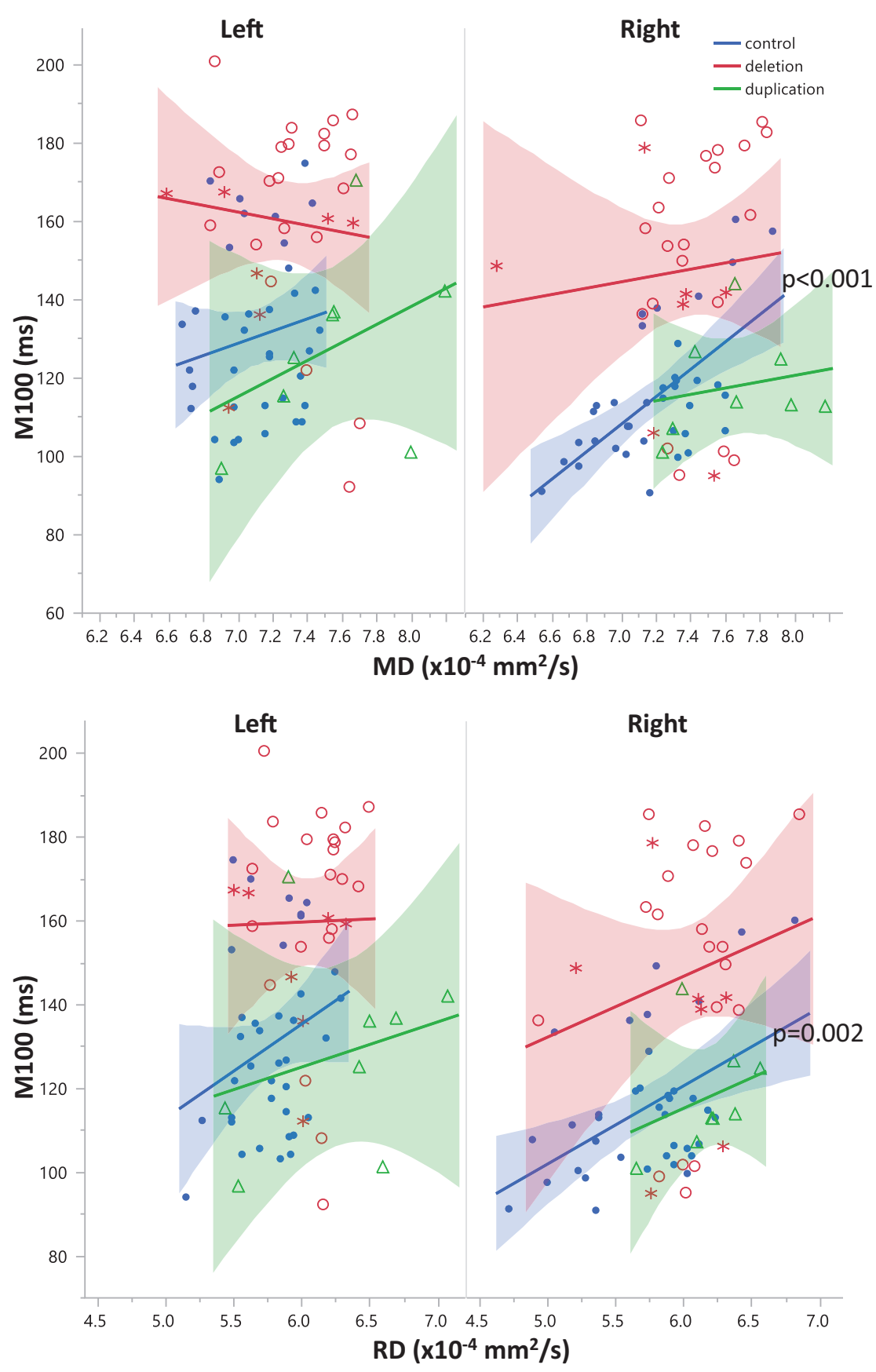

FIG 6. Relationships between diffusivity and M100 are shown for the left and right Heschl's gyri. While overall, there is a significant main effect of $\operatorname{MD}(P<.02)$ and $\operatorname{RD}(P<.001)$ on M100 latency, post hoc analysis within groups reveals that this is only significantly resolved for right hemisphere control MD and RD $(P<.001, P=.002$, respectively). Control subjects are blue, subjects with deletion are red, and subjects with duplication are green. Subjects with deletion and duplication with an ASD diagnosis are indicated by asterisks.

crease of right hemisphere auditory radiation MD would be necessary for an M100 increase of approximately $20 \mathrm{~ms}$. The $0.1 \times$ $10^{-4} \mathrm{~mm}^{2} / \mathrm{s}$ elevation of MD accounts for only a 2.5 -ms increase in M100, which is far short of the 20-ms delay observed in deletion carriers.

Both deletion and duplication groups have abnormal auditory system white matter microstructure; however, only the deletion group exhibited an M100 latency delay. Conduction velocity in the duplication group may indeed be facilitated given the small decrease in M100 latency observed in a prior study and repeated in the current study. ${ }^{13}$ These results strongly indicate that factors other than auditory radiation white matter integrity contribute to elongated M100 latency in deletion carriers and normal, or even possibly faster than typical, M100 latency in duplication carriers.

The entire auditory system is necessary for producing or eliciting a cortical response. The M100 integrates travel time through the cochlear nerve, brain stem, midbrain, thalamus, auditory radiation, and auditory cortex. Examination of factors other than auditory radiation white matter that modulate signal transmission velocity is warranted. Abnormal synapse development or neurotransmitter concentrations can introduce synaptic delays and abnormal patterns of action-potential generation. ${ }^{26,27}$ MR spectroscopy may be sensitive to changes in neurotransmitter levels. The organization of local networks within the auditory cortex could also affect the speed of the corticocortical looping, which is required for an evoked response. The quality of local corticocortical connectivity can be examined with MEG metrics such as phase amplitude coupling. ${ }^{28}$ Advanced diffusion MR imaging techniques that can discriminate crossing fibers may also be useful for examining the architecture of different cortical layers. ${ }^{29}$ One or more of these factors may be responsible for uncoupling structure from function in deletion and duplication carriers and delaying the M100 response in deletion carriers.

In controls, right hemisphere microstructure and M100 were more strongly and significantly correlated than in the left hemisphere. This result has been observed previously and possibly results from the hemispheric functional specialization of the superior temporal gyrus. ${ }^{30}$ In addition to auditory processing, the left superior temporal gyrus contains language networks. Thus, left hemisphere microstructural measures may reflect a mixture of auditory and language fibers, while the right hemisphere measures may primarily contain auditory fibers. Thus, right hemisphere auditory radiation diffusivity may be a proxy for diffusivity within all auditory system white matter.

\section{CONCLUSIONS}

White matter integrity is necessary but not sufficient for normal latency of the auditory evoked response. In carriers of the $16 \mathrm{p} 11.2$ 
$\mathrm{CNV}$, auditory radiation MD and RD were not strong modulators of M100 latency. The increased variance in the structure-function relationship indicates the influence of other factors that modulate electrophysiology. The pronounced M100 latency delay in $16 \mathrm{p} 11.2$ deletion carriers could only be partially explained by degraded white matter integrity. Compensatory mechanisms apparently overcome the abnormal white matter microstructure in 16 p11.2 duplication carriers to produce a normal (or possibly shorter) M100 latency. These results provide insight into the link among genetics, brain structure, function, and traits supporting neuropsychiatric disorders.

\section{ACKNOWLEDGMENTS}

We are grateful to all of the participating families and the Simons VIP Consortium. We appreciate obtaining access to phenotypic data on the SFARI Base. Approved researchers can obtain the Simons VIP population dataset by contacting SFARI. We are grateful to Thorsten Feiweier, $\mathrm{PhD}$, from Siemens for providing the 511C diffusion work-in-progress package.

Disclosures: Lisa Blaskey—RELATED: Grant: Simons Foundation. Emily KuschnerRELATED: Grant: Simons Foundation. * Pratik Mukherjee—RELATED: Grant: Simons Foundation*; Support for Travel to Meetings for the Study or Other Purposes: Simons Foundation. * Randy Buckner-RELATED: Grant: Simons Foundation, * Comments: A not-for-profit grant from the Simons Foundation supported portions of this work; Consultancy: Pfizer, Comments: paid consultant for Pfizer in the domain of neuroscience. Srikantan Nagarajan-RELATED: Grant: Simons Foundation.* Wendy K. Chung—RELATED: Grant: Simons Foundation. * Elliott H. Sherr-RELATED: Grant: Simons Foundation, ${ }^{\star}$ Comments: This work is part of a larger project, called Simons VIP, that is studying the biology of the chromosomal region, 16p11.2; Support for Travel to Meetings for the Study or Other Purposes: Simons Foundation. * Timothy P.L. Roberts—RELATED: Grant: Simons Foundation*; UNRELATED: Consultancy: Prism Clinical Imaging, Guerbet, Johnson \& Johnson. *Money paid to the institution.

\section{REFERENCES}

1. The Simons VIP Consortium. Simons Variation in Individuals Project (Simons VIP): a genetics-first approach to studying autism spectrum and related neurodevelopmental disorders. Neuron 2012; 73:1063-67 CrossRef Medline

2. Weiss LA, Shen Y, Korn JM, et al. Association between microdeletion and microduplication at 16p11.2 and autism. $N$ Engl J Med 2008;358:667-75 CrossRef Medline

3. Bochukova EG, Huang N, Keogh J, et al. Large, rare chromosomal deletions associated with severe early-onset obesity. Nature 2010; 463:666-70 CrossRef Medline

4. Jacquemont S, Reymond A, Zufferey F, et al. Mirror extreme BMI phenotypes associated with gene dosage at the chromosome 16p11.2 locus. Nature 2011;478:97-102 CrossRef Medline

5. Fernandez BA, Roberts W, Chung B, et al. Phenotypic spectrum associated with de novo and inherited deletions and duplications at 16 p11.2 in individuals ascertained for diagnosis of autism spectrum disorder. J Med Genet 2010;47:195-203 CrossRef Medline

6. Rosenfeld JA, Coppinger J, Bejjani BA, et al. Speech delays and behavioral problems are the predominant features in individuals with developmental delays and 16p11.2 microdeletions and microduplications. J Neurodev Disord 2010;2:26-38 CrossRef Medline

7. Hanson E, Bernier R, Porche K, et al; Simons Variation in Individuals Project Consortium. The cognitive and behavioral phenotype of the 16p11.2 deletion in a clinically ascertained population. Biol Psychiatry 2015;77:785-93 CrossRef Medline

8. Zufferey F, Sherr EH, Beckmann ND, et al; Simons VIP Consortium, 16 p11.2 European Consortium. A $600 \mathbf{~ k b}$ deletion syndrome at 16 p11.2 leads to energy imbalance and neuropsychiatric disorders. J Med Genet 2012;49:660-68 CrossRef Medline

9. Hanson E, Nasir RH, Fong A, et al; 16p11.2 Study Group Clinicians.
Cognitive and behavioral characterization of 16p11.2 deletion syndrome. J Dev Behav Pediatr 2010;31:649-57 CrossRef Medline

10. Qureshi AY, Mueller S, Snyder AZ, et al; Simons VIP Consortium. Opposing brain differences in 16p11.2 deletion and duplication carriers. J Neurosci 2014;34:11199-211 CrossRef Medline

11. Owen JP, Chang YS, Pojman NJ, et al; Simons VIP Consortium. Aberrant white matter microstructure in children with $16 \mathrm{p} 11.2$ deletions. J Neurosci 2014;34:6214-23 CrossRef Medline

12. Berman JI, Chudnovskaya D, Blaskey L, et al. Abnormal auditory and language pathways in children with 16p11.2 deletion. Neuroimage Clin 2015;9:50-57 CrossRef Medline

13. Jenkins J, Chow V, Blaskey L, et al. Auditory evoked M100 response latency is delayed in children with 16p11.2 deletion but not 16p11.2 duplication. Cereb Cortex 2015 Feb 11. [Epub ahead of print] CrossRef Medline

14. Roberts TP, Lanza MR, Dell J, et al. Maturational differences in thalamocortical white matter microstructure and auditory evoked response latencies in autism spectrum disorders. Brain Res 2013; 1537:79-85 CrossRef Medline

15. Lange N, DuBray MB, Lee JE, et al. Atypical diffusion tensor hemispheric asymmetry in autism. Autism Res 2010;3:350-58 CrossRef Medline

16. Berman J, Lanza M, Blaskey L, et al. High angular resolution diffusion imaging probabilistic tractography of the auditory radiation. AJNR Am J Neuroradiol 2013;34:1573-78 CrossRef Medline

17. Semel EM, Wiig EH, Secord W. Clinical Evaluation of Language Fundamentals (CELF-4). San Antonio: The Psychological Corporation; 2003

18. Elliott CD. Differential Ability Scales. 2nd ed. San Antonio: Pearson; 2007

19. Lord C, Risi S, Lambrecht L, et al. The autism diagnostic observation schedule-generic: a standard measure of social and communication deficits associated with the spectrum of autism. J Autism Dev Disord 2000;30:205-23 CrossRef Medline

20. Rutter M, Bailey A, Lord C. Social Communication Questionnaire (SCQ). Los Angeles: Western Psychological Services; 2003

21. Owen JP, Ziv E, Bukshpun P, et al. Test-retest reliability of computational network measurements derived from the structural connectome of the human brain. Brain Connect 2013;3:160-76 CrossRef Medline

22. van der Kouwe AJ, Benner T, Salat DH, et al. Brain morphometry with multiecho MPRAGE. Neuroimage 2008;40:559-69 CrossRef Medline

23. Berman JI, Chung S, Mukherjee P, et al. Probabilistic streamline q-ball tractography using the residual bootstrap. Neuroimage 2008; 39:215-22 CrossRef Medline

24. Fischl B, Sereno MI, Tootell RB, et al. High-resolution intersubject averaging and a coordinate system for the cortical surface. Hum Brain Mapp 1999;8:272-84 Medline

25. Roberts TP, Ferrari P, Stufflebeam SM, et al. Latency of the auditory evoked neuromagnetic field components: stimulus dependence and insights toward perception. J Clin Neurophysiol 2000;17:114-29 CrossRef Medline

26. Taschenberger H, von Gersdorff H. Fine-tuning an auditory synapse for speed and fidelity: developmental changes in presynaptic waveform, EPSC kinetics, and synaptic plasticity. J Neurosci 2000;20: 9162-73 Medline

27. Gaetz W, Bloy L, Wang DJ, et al. GABA estimation in the brains of children on the autism spectrum: measurement precision and regional cortical variation. Neuroimage 2014;86:1-9 CrossRef Medline

28. Canolty RT, Knight RT. The functional role of cross-frequency coupling. Trends Cogn Sci 2010;14:506-15 CrossRef Medline

29. Aggarwal M, Nauen DW, Troncoso JC, et al. Probing region-specific microstructure of human cortical areas using high angular and spatial resolution diffusion MRI. Neuroimage 2015;105:198-207 CrossRef Medline

30. Roberts TP, Khan SY, Blaskey L, et al. Developmental correlation of diffusion anisotropy with auditory-evoked response. Neuroreport 2009;20:1586-91 CrossRef Medline 